\title{
Zika virus outbreaks prompt warnings to pregnant women
}

\author{
Ingrid Torjesen
}

London

The rapid spread of Zika virus in the Americas, where there is little population immunity, is a major concern, especially given the possible link between infection during pregnancy and the congenital abnormality microcephaly, the World Health Organization has warned.

Margaret Chan, WHO's director general, said, “Although a causal link between Zika infection in pregnancy and microcephaly has not been established, the circumstantial evidence is suggestive and extremely worrisome. An increased occurrence of neurological syndromes, noted in some countries coincident with arrival of the virus, adds to the concern."

Travellers should stay informed about Zika virus and take measures to avoid being bitten by mosquitoes, particularly if they are pregnant, WHO has said, but it is not recommending restrictions on travel to affected countries, which include Brazil, Colombia, El Salvador, French Guiana, Guatemala, Haiti, Honduras, Martinique, Mexico, Panama, Paraguay, Puerto Rico, Suriname, and Venezuela.

Because of the possible heightened risk to pregnant women, health authorities in some countries, including the United Kingdom's National Travel Health Network and Centre and the US Centers for Disease Control and Prevention, are recommending that pregnant women reconsider plans to travel to affected countries. Meanwhile health authorities in at least five of the affected countries-Brazil, Colombia, Ecuador, El Salvador, and Jamaica — are advising women to avoid pregnancy for the moment.

Zika virus is transmitted by the same vector as the dengue virus-Aedes mosquito species_-and infection causes a mild illness that has symptoms not dissimilar to dengue fever but in which fever is lower and rash occurs earlier and more often.

The infection appeared in Africa decades ago where it stayed until the past decade, when it moved to Asia, across the Pacific, and now to South and Central America. WHO said that it expects the virus to spread to all countries in the Americas that have dengue carrying Aedes mosquitoes - that is, all except Canada and Chile.

Brazil has seen almost 4000 cases of microcephaly since October, which have been linked to the emerging Zika virus infection, for which there is no vaccine or treatment. Brazil saw its first case of Zika virus infection in May 2015, and cases have since been reported elsewhere in Latin America.

Experts in tropical diseases hope that the current outbreak of Zika virus infection in Latin America will behave in the same way as a rubella outbreak and subside once the pool of susceptible people has been exhausted. However, as with rubella, Zika virus infection in pregnant women is associated with congenital abnormalities, most notably microcephaly but also stillbirth and early neonatal death.

Laura Rodrigues, professor of infectious disease epidemiology at the London School of Hygiene and Tropical Medicine, who is involved in research into the high incidence of microcephaly in Brazil, told a press conference in London on 25 January that the "best hope" was that Zika virus infection would behave in the same way as rubella in a totally susceptible population.

"Most of the population gets it, most of the population becomes immune, many pregnant women have birth defects, and then it becomes a disease of childhood," she said. However, this would rely on longlasting natural immunity after infection, which has yet to be confirmed.

In the two major outbreaks in Asia and the Pacific-in Micronesia in 2007 and in French Polynesia in 2013-14) -Zika virus infection has behaved like rubella. In these cases outbreaks lasted around five months, and in Micronesia $80 \%$ of the population was exposed and in French Polynesia 50\%.

The aim, Rodrigues said, would be to develop a vaccine that could be given to girls in the same way as the rubella vaccine: before they get to an age where they get pregnant. In the meantime it would be "sensible" for women living in infected areas to postpone getting pregnant, and women who are pregnant, might be pregnant, or are considering pregnancy should "consider very carefully whether they want to go a place where there is a risk [of infection]," Rodrigues recommended.

Only a "narrow window" exists to gather data and set up and perform clinical trials during an outbreak, warned Trudie Lang, professor of global health research at the University of Oxford. "There has never been a clinical trial within a disease outbreak until Ebola."

The lack of research readiness for disease outbreaks in the UK and other countries was highlighted by the House of Commons Science and Technology Committee in its report on the handling of the epidemic of Ebola virus disease in west Africa. ${ }^{2}$

1 Dyer O. Jamaica advises women to avoid pregnancy as Zika virus approaches. BMJ 2016;352:i383.

2 O'Dowd A. Ebola outbreak exposed major weakness in UK handling of health emergencies. BMJ 2016;352:1455 
@) BMJ Publishing Group Ltd 2016 